NORDAFRIKA UND NAHOST IM KAMPF FUR NATIONALE UND SOZIALE BEFREIUNG 


\title{
STUDIEN ZUR GESCHICHTE
}

\section{ASIENS, AFRIKAS UND LATEINAMERIKAS}

\author{
Herausgegeben von
}

\section{WALTER MARKOV}

in Verbindung mit

Manfred Kossok und Lothar Rathmann

Band 19

Nordafrika und Nahost im Kampf

für nationale und soziale Befreiung 


\section{NORDAFRIKA UND NAHOST IM KAMPF FÜR NATIONALE UND SOZIALE BEFREIUNG}

A KA DEMIE - VERLAG - BERLIN 1968 
Erschienen im Akademie-Verlag GmbH, 108 Berlin, Leipziger Straße 3-4

Copyright 1968 by Akademie.Verlag GmbH

Lizenznummer : $202 \cdot 100 / 40 / 68$

Gesamtherstellung: VEB Druckerei „Thomas Müntzer", 582 Bad Langensalza

Bestellnummer: 2091/19 - ES 14 D/F

24,50 